\title{
A Technique to Improve Outcome of Silicone Finger Prosthesis
}

\author{
Rishabh Keshari ${ }^{1}$, Saumyendra V Singh ${ }^{2}$, Deeksha Arya $^{3}$, Mahimaa Gupta ${ }^{4}$
}

\begin{abstract}
Aim and objective: This technique aims to improve the outcome of silicone finger prostheses by reducing voids and the risk of tearing. Background: Obtaining an intact porosity-free silicone finger prosthesis is a difficult task owing to the high viscosity of the material, coupled with difficult packing and retrieval in the thin ventral part of the mold (which is overlapped by the stump model).

Technique: This technique describes predictable packing of silicone along with easier retrieval of prosthesis and mold. Incorporation of brass dowel pins in the stump model just before investment and packing of mold helps in preventing mold/stump fracture and tearing of silicone finger prosthesis.

Conclusion: The described technique helps in creating a silicone finger prosthesis with fewer porosities, which can be easily extricated from the mold, thereby also increasing the longevity of the mold.

Keywords: Dowel pin, Finger amputation, Finger prosthesis, Medical-grade silicone, Mold, Packing, Porosities. International Journal of Prosthodontics and Restorative Dentistry (2021): 10.5005/jp-journals-10019-1323
\end{abstract}

\section{INTRODUCTION}

The fabrication of finger prostheses is an effective approach in the rehabilitation of patients with complete or partial finger amputation. ${ }^{1}$ Ideal finger prosthesis should be lifelike with realistic surface detailing, close skin shade matching, thin margins merging with the skin, incorporation of fingernails, wrinkles, and pigmentations. ${ }^{2,3}$ For this, prostheses made from medical grade silicone ${ }^{4}$ should be condensely packed into a two-piece mold, without voids and porosities, to obtain the exact finishing and margin of the prosthesis. This is a difficult task with viscous silicone ${ }^{5}$ especially in crevice areas between stump and mold. This paper describes a technique to help overcome this difficulty, facilitate prosthesis retrieval without tearing of margins, and prevent mold fracture/breakage.

\section{TECHNIQUe}

A 24-year-old female patient reported to the Department of Prosthodontics, with a chief complaint of partially missing index finger of the right hand. History revealed that the patient lost this part 5 years back due to a traumatic injury. On examination, amputation was above the mid part of the middle phalanx. There was no pain on palpation, and the surrounding skin showed no signs of inflammation and infection. The patient had no history of the previous prosthesis. The treatment options included implantsupported finger prosthesis and suction retained conventional silicon prosthesis. The patient was unwilling for any surgical procedure, so the fabrication of a conventional silicone prosthesis was planned.

\section{Impression Making}

A thin layer of petroleum jelly was applied to the patient's hand before making an impression to prevent impression material from adhering to the tissue surface. A cylindrical box was made using modeling wax (Maarc, Maharashtra, India) according to the size of the amputated finger (Fig. 1A). Impressions of the affected digit

\footnotetext{
${ }^{1-4}$ Department of Prosthodontics, King George's Medical University, Lucknow, Uttar Pradesh,

Corresponding Author: Saumyendra V Singh, Department of Prosthodontics, King George's Medical University, Lucknow, Uttar Pradesh, India, Phone: +91 9415470702, e-mail: saumyendravsingh@ rediffmail.com

How to cite this article: Keshari R, Singh SV, Arya D, et al. A Technique to Improve Outcome of Silicone Finger Prosthesis. Int J Prosthodont Restor Dent 2021;11(3):142-145.

Source of support: Nil

Conflict of interest: None
}

were made with irreversible hydrocolloid impression material (Zelgan, North Carolina, USA) while asking the patient to place her finger in a relaxed state in the selected cylindrical tray without
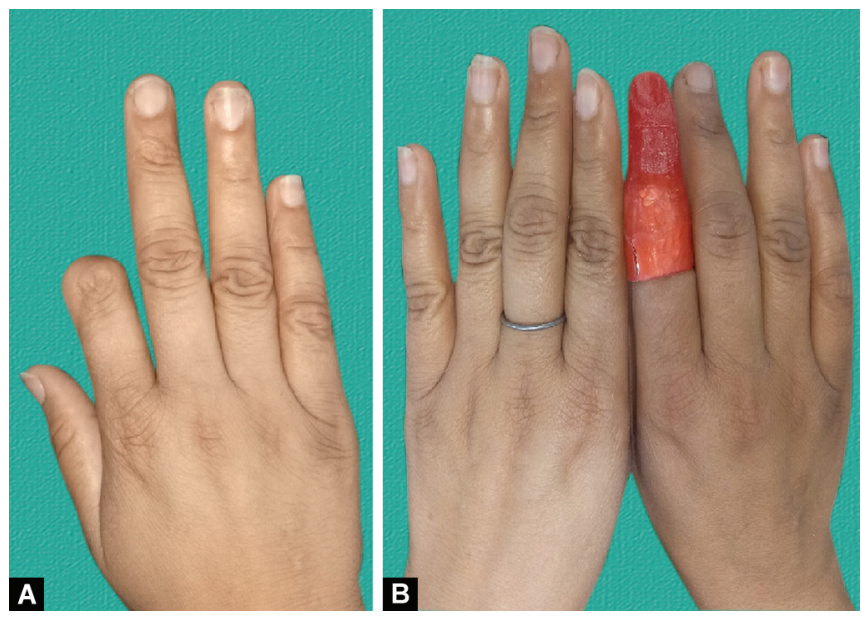

Figs $1 A$ and $B$ : (A) Amputated digit; (B) Wax pattern try-in

(c) The Author(s). 2021 Open Access This article is distributed under the terms of the Creative Commons Attribution 4.0 International License (https://creativecommons. org/licenses/by-nc/4.0/), which permits unrestricted use, distribution, and non-commercial reproduction in any medium, provided you give appropriate credit to the original author(s) and the source, provide a link to the Creative Commons license, and indicate if changes were made. The Creative Commons Public Domain Dedication waiver (http://creativecommons.org/publicdomain/zero/1.0/) applies to the data made available in this article, unless otherwise stated. 
touching its bottom. The impression was then poured with ADA type IV dental stone (Kal Rock, Kala Bhai Karson Pvt, Mumbai, India) to create a positive replica/stump model of the amputated finger and associated structures.

\section{Wax Pattern Fabrication}

Another impression of the contralateral normal digit in a relaxed state was made using irreversible hydrocolloid impression material (Zelgan, North Carolina, USA) in a cylindrical box tray similar to the one described above. Modeling wax (Maarc, Maharashtra, India) was melted and poured into the impression to obtain a wax duplicate of the normal finger. Then, the middle phalanx area of the stump model was reduced by $0.5 \mathrm{~mm}$ using a flame-shaped tungsten carbide bur and BP blade no. 23, to produce a snugly fitting finger prosthesis.

The wax pattern of a contralateral finger was gradually seated over the prepared finger stump, and necessary modifications in thickness and anatomy were done. Surface characterization (skin folds and wrinkles) was incorporated to reproduce the exact anatomy of a missing finger. The pattern was assessed and further adjusted keeping in mind alignment, size, shape, and contours of the contralateral finger. Wax pattern trial was done for fit and aesthetics (Fig. 1B). Finally, the wax pattern was re-marginated on the stump model.

\section{Stump Model Preparation}

A single sheet of tin foil (Buffalo Dental Manufacturing Co Inc., New York, USA) was adapted distal to the wax pattern margin over the model (Fig. 2). This is important for creating a slight space between model and mold, which would help in the easier removal of the stump model from the mold in the future. Two die pin dowels (HKAA, China) were attached at the distal end of the ventral aspect of the stump model, each at a separation of $5 \mathrm{~mm}$ from each other and from the wax pattern margin. It is important to ensure that the dowel pins emerge perpendicular to the stump model and are parallel to each other (Fig. 2). Modeling wax (Maarc, Maharashtra, India) was adapted in a round shape over the open end of each dowel (Fig. 2) to allow easier tracing of the tips of the dowel. These dowel tips can be pushed out later to aid stump removal.

\section{Flasking and Processing of Finger Prosthesis}

A rectangular box of $15 \times 8 \times 8 \mathrm{~cm}$ dimension was made using modeling wax (Maarc, Maharashtra, India). The first pour of the mold

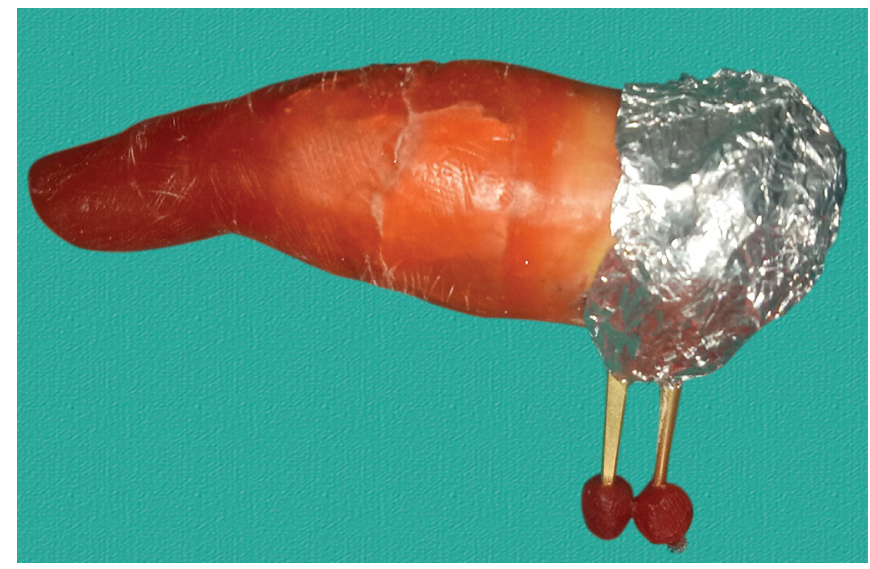

Fig. 2: Die pin dowels attached was done into this box using type IV white dental stone (Kalabhai Karson Pvt Ltd., Mumbai). The wax pattern on the stump model was invested into the first pour before it began to set, ensuring that the ventral side went into this part of the mold till the junction of dorsal and ventral halves of the pattern (Fig. 3). After setting the first pour, four triangular indices were trimmed into each side and separating media (Cold Mold Seal, Pyrax, Roorkee, India) was applied to complete the second and final pour. Dewaxing was done routinely and two halves of the mold are separated. The stump model was removed and checked for reseating (Fig. 4).

\section{Finishing and Delivery of Prostheses}

Shade matching was done in the presence of a patient using a spectrophotometer (E-Skin, Spectromatch Ltd., Bath, UK). Intrinsic colors were mixed in the silicone to achieve appropriate characterization for palmar, dorsal, and nail surfaces. The stump model was removed from the mold and material for the palmar surface was packed (Fig. 5A), followed by reseating of the stump model (Fig. 5B) and remaining material packing (Fig. 5B). The material was cured and polymerized as per the manufacturer's instructions. The stump model was easily removed from the mold (Fig. 6A) by pushing in the dowel tips and the prosthesis was gently teased off the stump model, without tearing. Excess material was cut using a scissor and extrinsic staining was done.

A customized open-ended metal ring was used as an auxiliary retention aid and served the secondary purpose of masking prosthesis-skin junction (Fig. 6B). The patient was educated about the use and maintenance of the prosthesis and recalled at trimonthly intervals for follow-up.

\section{Discussion}

Silicone has texture and flexibility similar to skin and helps make a comfortable prosthesis with the capacity for camouflaging skinprosthesis margin. The thinness of the silicone prosthesis allows good sensibility through it. ${ }^{6}$ Also, the snug fit of this prosthesis over the amputated digit provides a massaging and desensitizing effect. However, the presence of unsightly voids in the prosthesis is common, as silicone is viscous and does not flow easily into inaccessible areas, ${ }^{1}$ especially where the mold and stump model are in close approximation. To overcome this problem, a technique has been suggested wherein die pins are attached to the stump model

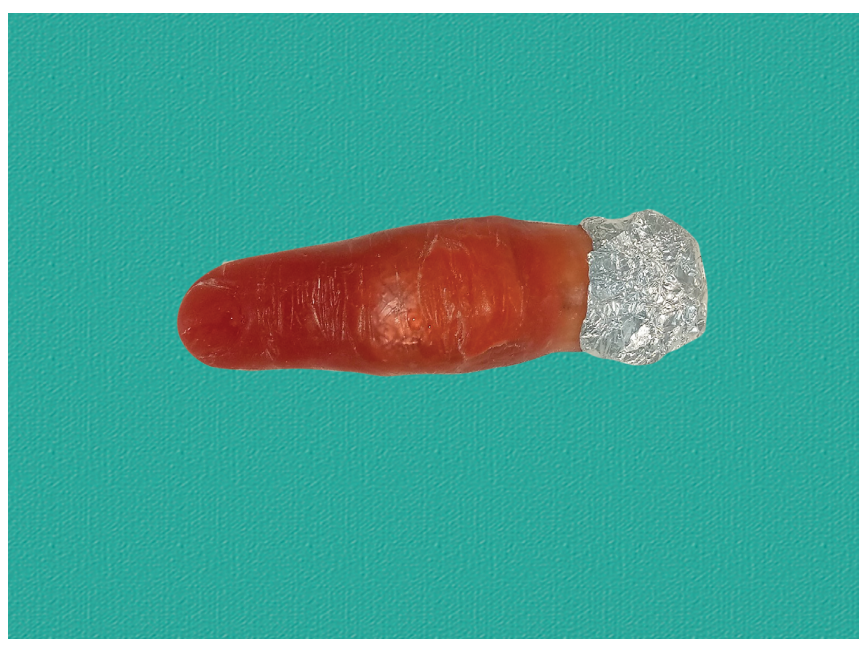

Fig. 3: First pour with pattern invested 

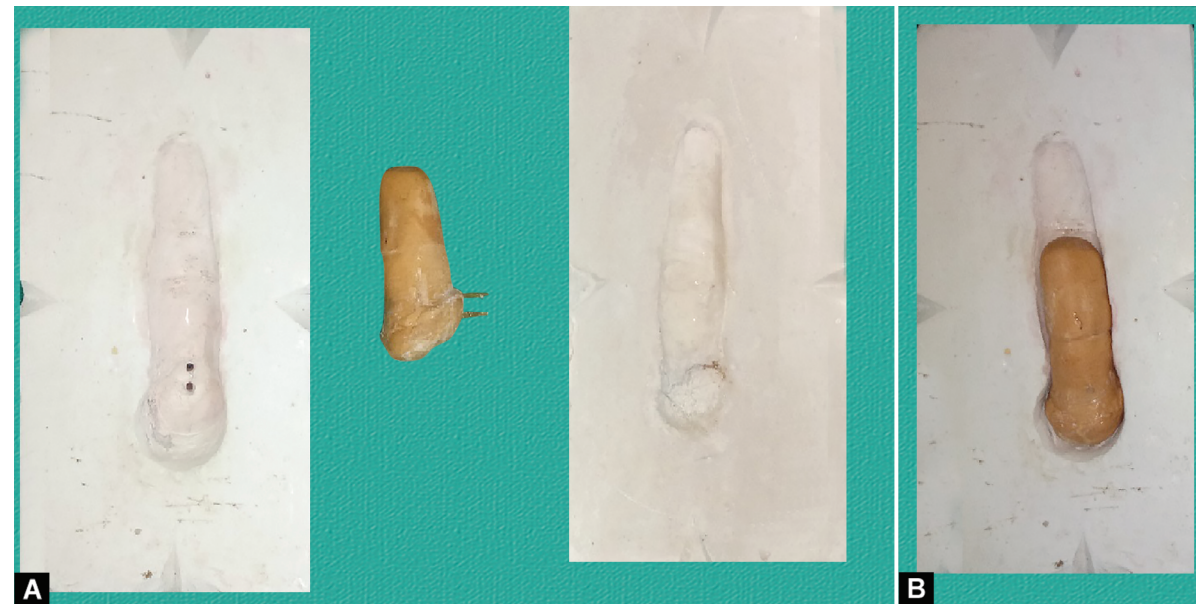

Figs 4A and B: Two halves of the mold separated after dewaxing: (A) Stump removed; (B) Re-seated

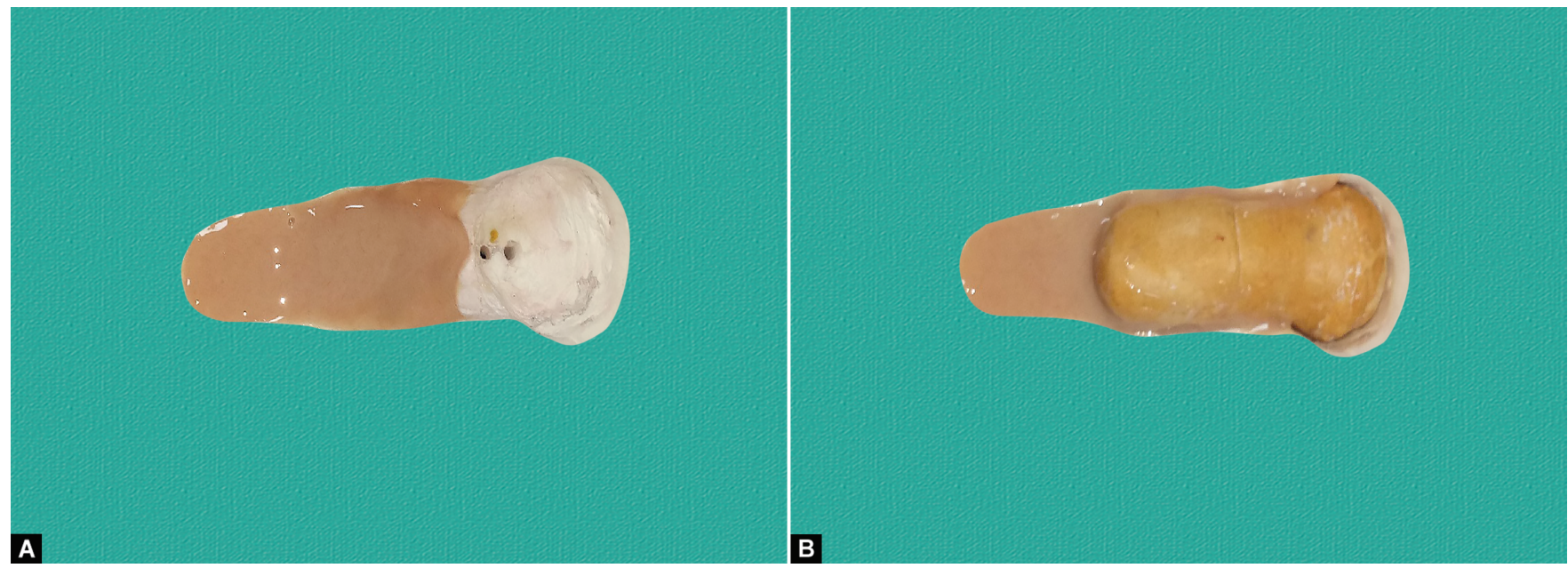

Figs 5A and B: Layering silicone on the ventral side: (A) Stump removed; (B) Re-seated

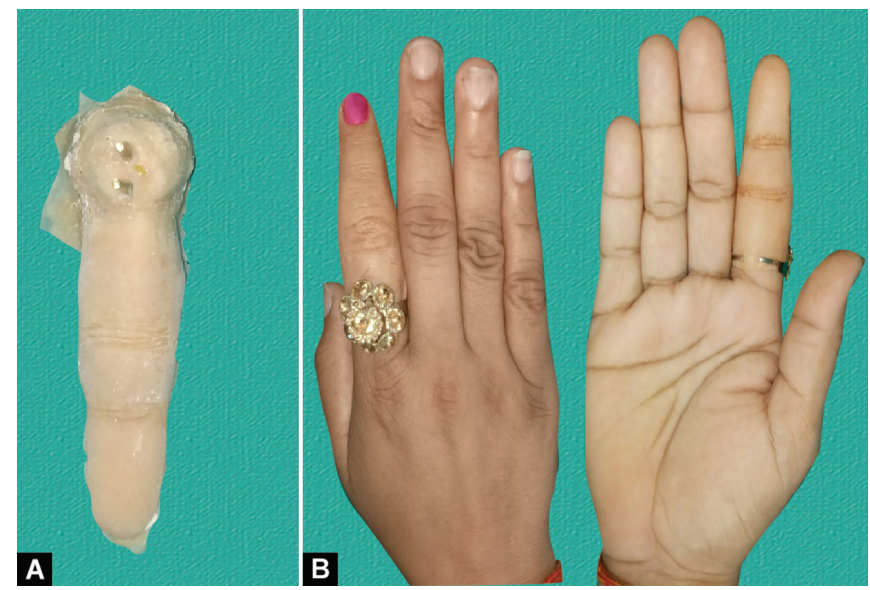

Figs $6 \mathrm{~A}$ and B: Prosthesis: (A) As retrieved from the mold; (B) At the time of delivery

before the investment of the pattern. This technique allows three advantages over conventional investment technique: (i) adequate flow of silicone material into narrow spaces between the digit and mold, with less incidence of porosities, (ii) easy retrieval and precise replacement of the stump model into the invested mold (which often fractures to remove the prosthesis), and (iii) easy extrication of the prosthesis without tearing. A disadvantage of the technique includes added laboratory steps in the form of tin foil adaptation and dowel pin placement.

Thomas ${ }^{7}$ has described an alternate method of mold preparation to facilitate easy layering of silicone between the mold and stump model, also facilitating easy removal without trapping. Here, undercuts were created in the stump model itself that locked onto the two mold parts. This was similar to our technique in as much, that both lead to easy separation of the model from the two mold halves. However, our technique would possibly create a more accurate and easy adaptation of the different components as a result of the metal-dental stone (compared with dental stone-dental stone) interface. In another study, Shari et al. used a three-piece mold technique with the help of a die-pin system to make their prosthesis hollow and more comfortable. ${ }^{8}$

\section{Conclusion}

The described technique helps in creating a silicone finger prosthesis with fewer porosities, which can be easily extricated from the mold, thereby also increasing the longevity of the mold. 


\section{References}

1. Kini AY, Byakod PP, Angadi GS, et al. Comprehensive prosthetic rehabilitation of a patient with partial finger amputations using silicone biomaterial: a technical note. Prosthet Orthot Int 2010;34(4):488-494. DOI: 10.3109/03093646.2010.486391.

2. Pattanaik B, Pattanaik S. Fabrication of a functional finger prosthesis with simple attachment. J Indian Prosthodont Soc 2013;13(4):631-634. DOI: 10.1007/s13191-012-0228-4.

3. Tripathi S, Singh RD, Chand P, et al. A modified approach of impression technique for fabrication of finger prostheses. Prosthet Orthot Int 2012;36(1):121-124. DOI: 10.1177/0309364611431480.
4. Huber H, Studer SP. Materials and techniques in maxillofacial prosthodontic rehabilitation. Oral Maxillof Surg Clin 2002;14(1):73-93. DOI: 10.1016/s1042-3699(02)00018-3.

5. Mitra A, Choudhary S, Garg H. Maxillofacial prosthetic materials - an inclination towards silicones. J Clin Diagn Res 2014;8(12):8-13. DOI: 10.7860/JCDR/2014/9229.5244.

6. Parry CB. The rehabilitation of the hand. J Aust Occup Ther 1959;6:35.

7. Thomas KF. The art of clinical anaplastology. SThomas 2006; 135-137.

8. Shari SR, Harshakumar K, Janardanan. K. Fabrication of a hollow finger prosthesis using a three-piece mould technique: a case report. Int J Appl Dent Sci 2021;7(2):370-373. DOI: 10.22271/oral.2021.v7.i2f. 1233. 